\title{
Hydrophilic intraocular lens opacification after posterior lamellar keratoplasty - a material analysis with special reference to optical quality assessment
}

\author{
Bert C. Giers ${ }^{1 \dagger} \mathbb{D}$, Tamer Tandogan ${ }^{1 \dagger}$, Gerd U. Auffarth ${ }^{1}$, Chul Y. Choi ${ }^{2}$, Florian N. Auerbach ${ }^{1}$, Saadettin Sel ${ }^{1}$, \\ Christian Mayer ${ }^{3}$ and Ramin Khoramnia ${ }^{1 *}$
}

\begin{abstract}
Background: Laboratory analysis and optical quality assessment of explanted hydrophilic intraocular lenses (IOLs) with clinically significant opacification after posterior lamellar keratoplasty (DMEK and DSAEK).

Methods: Thirteen opacified IOLs after posterior lamellar keratoplasty, 8 after descemet stripping automated endothelial keratoplasty (DSAEK), 3 after descemet membrane endothelial keratoplasty (DMEK) and 2 after both DSAEK and DMEK were analysed in our laboratory. Analyses included optical bench assessment for optical quality, light microscopy, scanning electron microscopy (SEM) and energy dispersive X-Ray spectroscopy (EDS).

Results: In all IOLs the opacification was caused by a thin layer of calciumphosphate that had accumulated underneath the anterior optical surface of the IOLs in the area spared by the pupil/anterior capsulorhexis. The calcifications lead to a significant deterioration of the modulation transfer function across all spatial frequencies of the affected IOLs.

Conclusions: The instillation of exogenous material such as air or gas into the anterior chamber increases the risk for opacification of hydrophilic IOLs irrespective of the manufacturer or the exact composition of the hydrophilic lens material. It is recommended to avoid the use of hydrophilic acrylic IOLs in patients with endothelial dystrophy that will likely require procedures involving the intracameral instillation of air or gas, such as DMEK or DS(A)EK.
\end{abstract}

Keywords: Cornea, Fuchs endothelial dystrophy, Posterior lamellar keratoplasty, Descemet membrane endothelial keratoplasty, Cataract surgery, Intraocular lens, Optical quality, Complications, IOL explantation

\section{Background}

The opacification of hydrophilic acrylic intraocular lenses (IOLs) is a rare complication, usually occurring in the late postoperative period [1]. The exact causes and pathomechanisms leading to hydrophilic IOL opacification are unknown. There have been sporadic reports about high incidences of IOL opacification affecting whole batches of IOLs of individual manufacturers irrespective of secondary surgical interventions or

\footnotetext{
* Correspondence: Ramin.Khoramnia@med.uni-heidelberg.de

${ }^{\dagger}$ Equal contributors

${ }^{1}$ International Vision Correction Research Centre and the David J. Apple

International Laboratory for Ocular Pathology, Department of

Ophthalmology, University of Heidelberg, INF 400, 69120 Heidelberg,

Germany

Full list of author information is available at the end of the article
}

comorbidities [2-4], so that material impurities and faulty manufacturing or storage as well as interactions with the packaging material have been suggested as causative factors in these cases. Individual factors, such as ocular inflammation or ocular and systemic comorbidities that affect ocular metabolism, may contribute to the process $[2,3]$.

Secondary surgical interventions with instillation of foreign material into the anterior chamber, such as air, gas or rtPA [5] seem to increase the risk of IOL opacification. In particular, there has been an increasing number of reports since 2011/2012 about hydrophilic IOL opacification following posterior lamellar keratoplastic surgery involving the intracameral instillation of air or gas [6-13]. In all of 


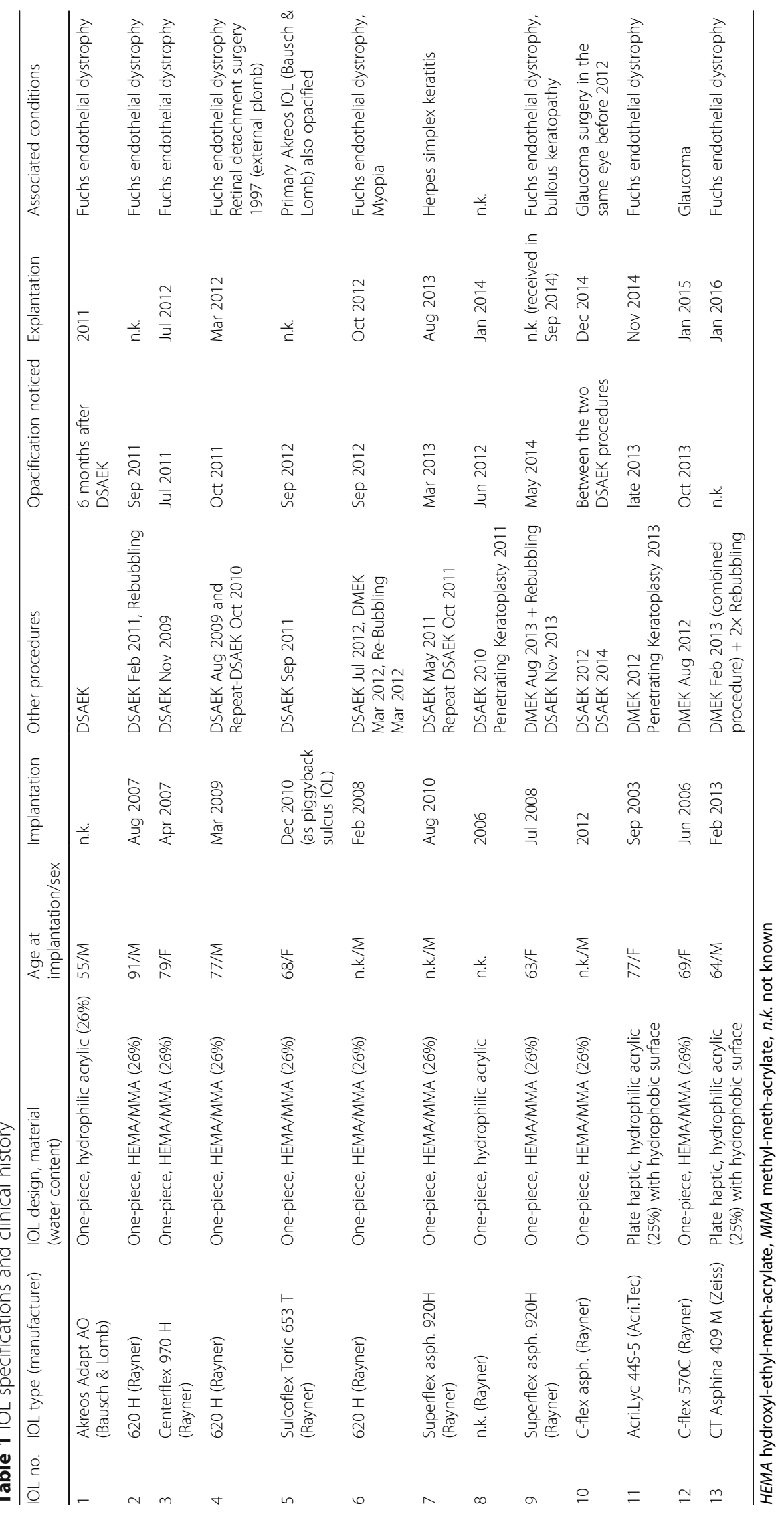


these cases, the opacification was limited to a more or less circular area of the anterior optical surface, corresponding to the zone of contact with the instilled air or gas.

Opacifications of the IOL optic may cause reduced visual acuity, decreased contrast sensitivity and glare [3]. In clinically significant IOL opacifications an IOL explantation is the only treatment option. IOL explantation procedures are, however, often associated with an increased complication rate [14].

We report on the laboratory examination of 13 opacified IOLs which were explanted due to reduced visual acuity after posterior lamellar keratoplasty. All IOLs were examined using light microscopy, scanning electron microscopy and X-ray spectroscopy. In those specimen, that were explanted in toto, the optical properties were examined at an optical bench using modulation transfer function (MTF) measurements and documenting United States Air Force (USAF) 1951 resolution target images. To our knowledge, this is the largest series of opacified IOL explants, that systematically analyses not only the nature of the IOL opacification using light and electron microscopy as well as elementary analysis, but adds an analysis of the explanted IOLs' optical properties using an optical bench. It therefore provides an objective measure to the clinical findings of visual deterioration and further adds to our understanding of IOL opacification after posterior lamellar keratoplasty procedures.

\section{Methods}

IOL specifications, handling and gross examination

A total of 13 IOLs that had been explanted due to clinically significant opacification after posterior lamellar keratoplasty procedures were examined at the David J Apple International Laboratory for Ocular Pathology at the Department of Ophthalmology of the University of Heidelberg. In 8 subjects the opacification had appeared after descemet stripping automated endothelial keratoplasty (DSAEK; IOLs $1-5,7,8,10$ ) and 3 subjects had a descemet membrane endothelial keratoplasty (DMEK; IOLs 11-13) performed prior to the occurrence of IOL opacification. In two cases (IOLs 6 and 9), the patients had first received a DMEK and in the later course a DSAEK in the same eye prior to IOL opacification. IOL specifications, patient data and clinical history (as far as available) are summarized in Table 1. For shipment, the explanted IOLs were submerged in isotonic sodiumchloride solution. After gross macroscopic inspection, the IOLs were examined using an Olympus BX50 light microscope (Olympus Optical Co. Ltd., Tokyo, Japan). Photos were acquired using an Olympus C-7070 Camera (Olympus Optical Co. Ltd., Tokyo, Japan).

\section{Assessment of optical quality}

IOLs that were explanted in toto were tested using an optical bench (OptiSpheric IOL Pro, Trioptics, Germany) for

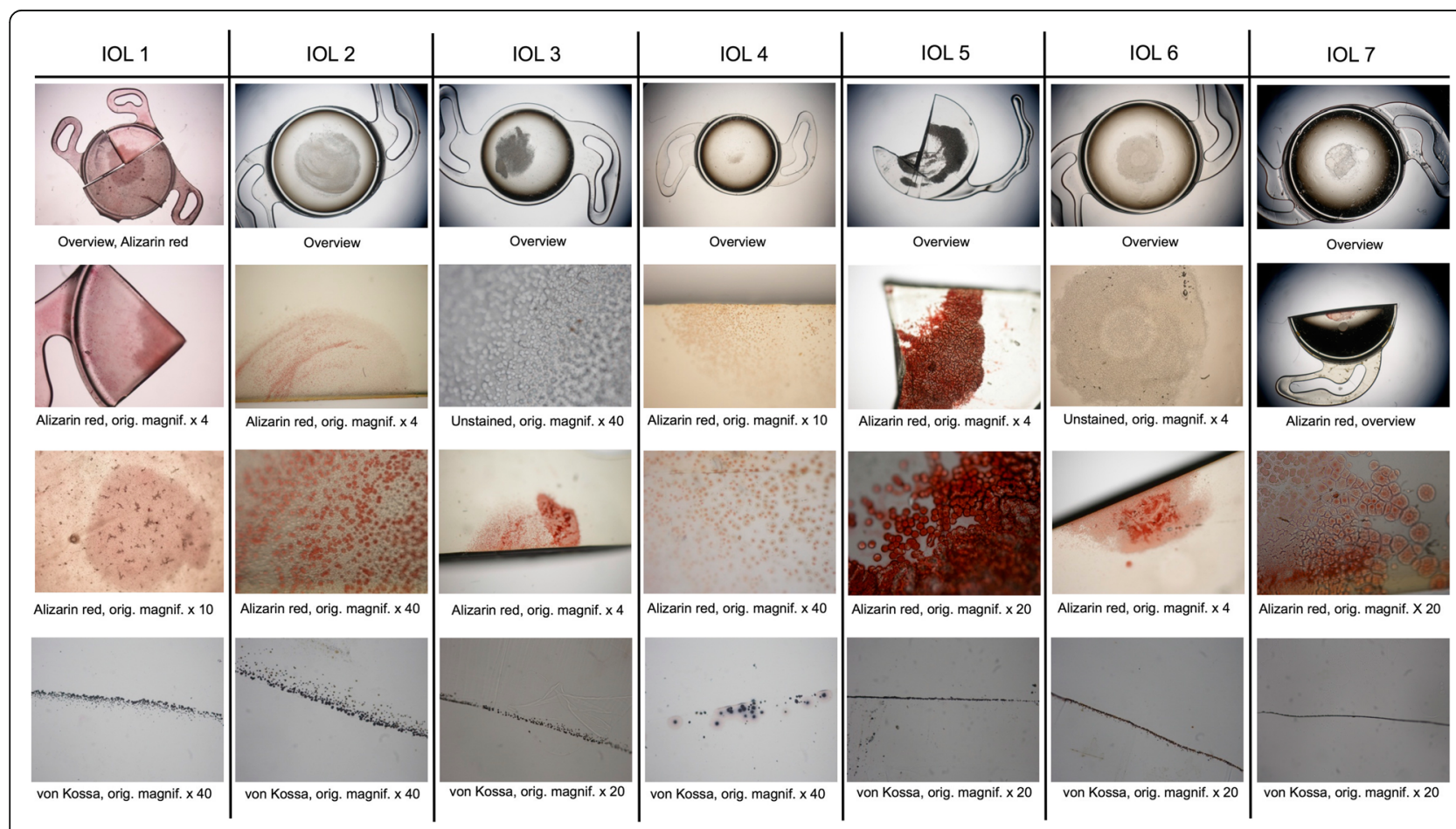

Fig. 1 IOLs 1-7. Light microscopy images representing an overview of the IOL optic and higher magnification images with Alizarin Red and von Kossa stainings. Details and magnification as noted under each individual panel 
optical quality according to a protocol described in ISO norm 11-979-2. Optical quality parameters assessed included the modulation transfer function (MTF), which describes the resolution of an optical system as the ratio of relative image contrast to object contrast. The optical bench features different types of targets as objects which are projected to the infinity through a collimator. The tested lens therefore creates an image of the target object at its focal plane. The light source illuminating the target is a broad band visible spectrum light source associated with a narrow band interferential filter at $546 \mathrm{~nm}$. The measurement head composed of a microscope objective and an imaging system conjugated with a CCD camera scans through the imaging zone to find the best focus image created by the tested IOL. The microscope objective relays the image obtained via the IOL to the CCD, and the system's software analyises the output of the CCD to create the MTF curve. For visualisation purposes United States Air Force (USAF) 1951 resolution targets were used to compare the image quality at the focal plane.

\section{Light microscopy}

After optical measurement, all IOLs were split in halves and one half was analysed by light microscopy using the
Alizarin Red and von Kossa stainings. For Alizarin Red staining, the IOL was fixated for two minutes in $4 \%$ buffered formaldehyde solution and rinsed with distilled water. Thereafter, the IOLs were submerged in $1 \%$ Alizarin Red solution for $3 \mathrm{~min}$, rinsed again and were then examined using a light microscope. The specimen were then dehydrated and embedded in paraffin. Five micrometers vertical sections through the optical center of the IOLs were taken and subsequently stained using the von Kossa method. Sections were deparaffinized, rehydrated and incubated in 5\% silver nitrate, treated with UV light for $30 \mathrm{~min}$ and rinsed several times. After incubation with $5 \%$ sodiumthiosulfate and a final rinsing step, sections were analysed using an Olympus BX50 light microscope equipped with a camera (Olympus C7070, Olympus Optical Co. Ltd., Tokyo, Japan).

\section{Scanning electron microscopy and energy dispersive $\mathrm{X}$-ray spectroscopy}

The second halves of the explanted IOLs were sent to the Max-Planck-Institute for Polymer Research in Mainz, Germany, for further analysis including scanning electron microscopy (SEM) and energy dispersive X-Ray spectroscopy (EDS). For SEM analysis $2.5 \mu \mathrm{m}$ cross

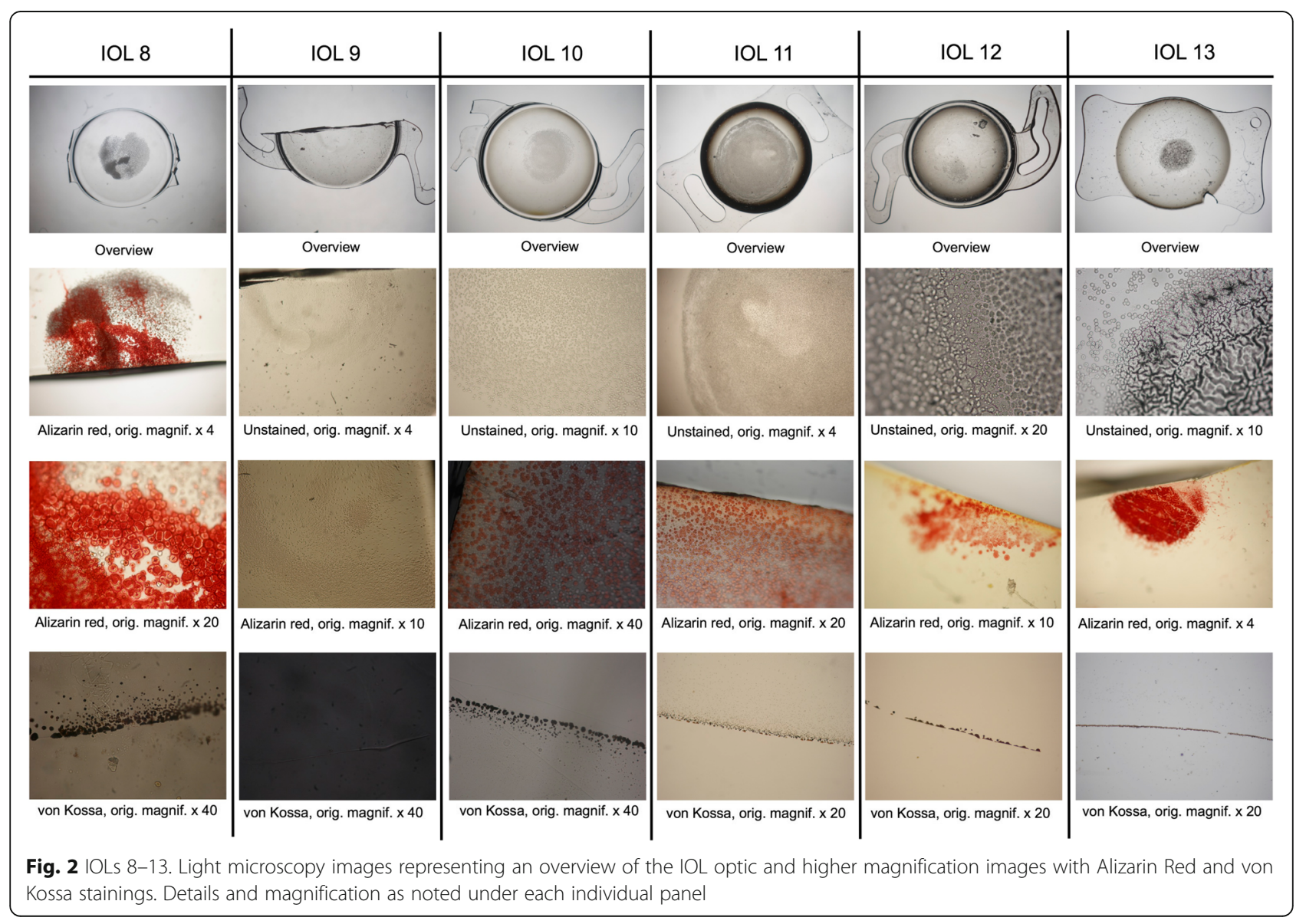


sections through the IOL material were acquired using an ultramicrotome (UCT, Leica, Germany) and a $35^{\circ}$ diamond knife (Diatome, Switzerland) and mounted on silicium grids. SEM examinations were carried out in low voltage $(<1 \mathrm{kV})$ conditions using an SU8000 microscope (Hitachi, Japan). For a local chemical analysis EDS was performed using a Quantax 400 EDS detector (Bruker, Germany) to detect exogenous chemical elements within the IOL material.

This study solely involves laboratory analyses of IOL explants. No additional procedures on humans or animals were performed. An informed consent and ethics committee approval were therefore not required.

\section{Results}

Macroscopically, all IOLs showed a more or less circular opacification of the central anterior optical surface, sparing the peripheral optical zone and the haptics (Figs. 1 and 2). Light microscopy revealed numerous and sometimes confluent ovaloid or spherical deposits just underneath the anterior optical surface. The opacifications invariably stained positive with Alizarin Red for calcium. The von Kossa staining of cross sections of the IOLs revealed numerous fine granular crystalline deposits distributed in a line parallel to and immediately underneath the anterior optical surface of all IOLs. The posterior surfaces as well as the haptics of all examined IOLs were free of deposits.

SEM revealed numerous fine and roughly spherical crystalline deposits of approx. $2-15 \mu \mathrm{m}$ in diameter that were situated immediately underneath the optical surface of the IOL, often causing micro-distortions of the optical surface (Fig. 3). In some cases there appeared to be microvacuoles within the crystals causing microscopically small cracks or openings through the optical surface (Fig. 3, IOLs 4 and 9). Element mapping (Fig. 4) and $\mathrm{X}$-ray spectroscopy (Fig. 5) showed, that the deposits are made up of calcium phosphate.

MTF measurements of those IOLs that were received with an intact optic (IOLs No. 2-4, 6-8 and 10-13) showed a significant decrease in optical quality with MTF values deteriorated at all spatial frequencies (Fig. 6a). The

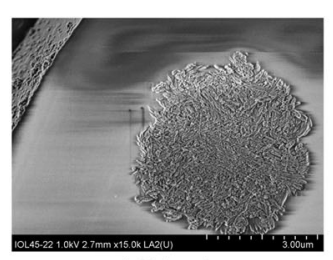

IOL 1

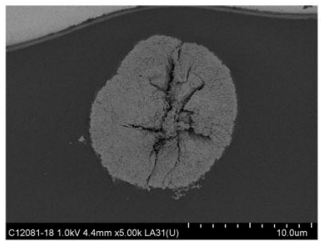

IOL 4

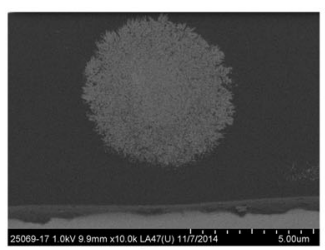

IOL 7

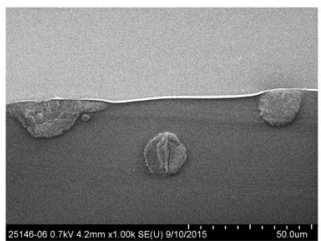

IOL 10

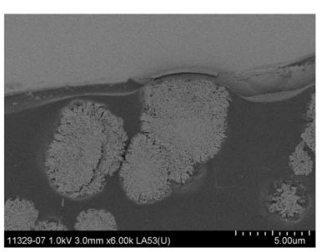

IOL 2

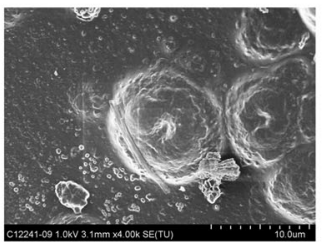

IOL 5

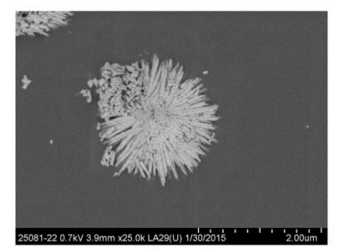

IOL 8



IOL 11

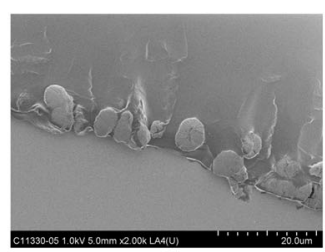

IOL 3

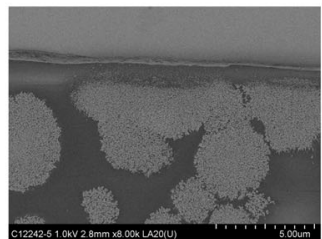

IOL 6

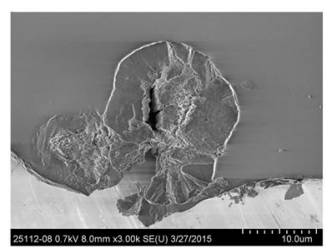

IOL 9

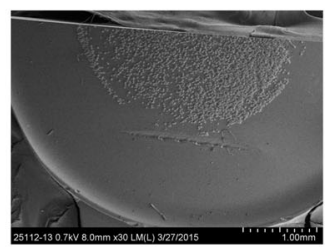

IOL 12

Fig. 3 SEM-scans from cross-sections through the anterior optical surface (IOL 1-4 and 6-11), high magnification scan of the opacified anterior optical surface showing individual calcium deposits (IOL 5), low magnification overview of an IOL specimen showing the opacifications in the center of the anterior optical surface (IOL 12). Magnification and scale bar given in each individual panel 


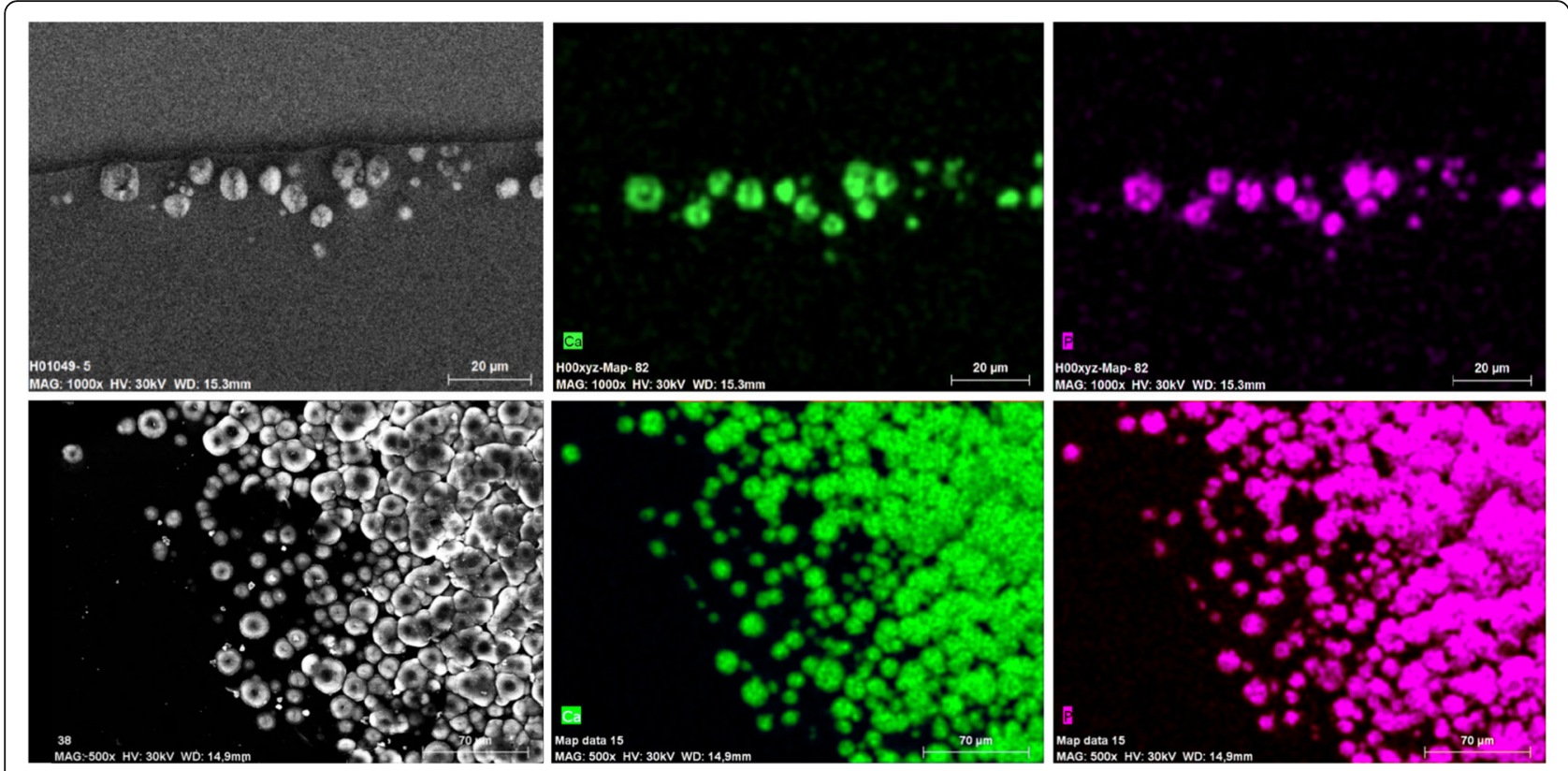

Fig. 4 Exemplary Element Mapping showing calcium (green) and phosphorus (magenta) within the deposits in IOL 5

analysis of USAF target images illustrates a significant loss in brightness and contrast, however, with light adjustment an acceptable image resolution could be achieved (Fig. 6b,c).

\section{Discussion}

Since the advent of minimally invasive keratoplastic surgery, particularly DS(A)EK and DMEK, there has been an increasing number of reports on postoperative opacification of hydrophilic acrylic IOLs [6-13, 15-18]. These techniques commonly rely on the instillation of an air or gas-bubble into the anterior chamber to foster the adhesion of the graft tissue with the posterior corneal surface $[19,20]$. Whereas in previous reports on IOL opacifications not associated with secondary surgical interventions the whole optical surface (including the posterior surface) of the IOL and in some cases even the haptics showed signs of opacification [2, 3], suggesting material impurities or faulty manufacturing, the opacification pattern observed in hydrophilic IOLs after posterior lamellar keratoplasty is very distinct. The opacified area in these cases is usually limited to a more or less circular area of the anterior optical surface, which usually corresponds to the area of the pupil and/or anterior capsulorhexis, thus to the zone of contact with the instilled air or gas, whereas parts of the IOLs covered by the capsular bag, including the haptics and the posterior optical surface, displayed no sign of opacification $[6-11,13,15,16]$. Furthermore, it appears to equally affect hydrophilic IOLs from different manufacturers (Bausch \& Lomb [6, 13], Ciba Vision [11, 13],
Lenstec [17], Oculentis [10, 13], Rayner [8, 17], Acri.Tec [16], Zeiss [18]). It seems, that the instillation of exogenous material into the anterior chamber and its prolonged direct contact to the IOL's surface increases the risk of opacification of hydrophilic acrylic IOLs irrespective of the manufacturer and the exact material composition.

It should be noted at this point, that the high amount of Rayner IOLs in this study is predominantly owed to the circumstance, that Rayner Intraocular Lenses Ltd. routinely send their IOLs to our laboratory for pathological analysis, whereas IOLs from other manufacturers only reach us through individual cooperating ophthalmic surgeons. The many reports on IOL calcification after posterior lamellar keratoplasty clearly point to a general problem with the hydrophilic acrylate material, rather than a problem with IOLs from certain individual manufacturers.

To the best of our knowledge, this is the largest series of opacified IOLs after posterior lamellar keratoplasty analysed to date. Our results show, that the opacification of the analysed IOLs is caused by crystalline deposits underneath the anterior optical surface, which stain positively with Alizarin Red and the von Kossa method, suggesting a high calcium content. Energy dispersive X-ray spectroscopy for elementary analysis of the composition of the deposits proves, that the deposits consist of calcium phosphate. This confirms the findings of other groups, who demonstrated calcium phosphate deposits in explanted IOLs using similar methods $[2,3,7,10,13,18,21]$.

The calcification-pattern occurring after posterior lamellar keratoplasty as described here and in other 


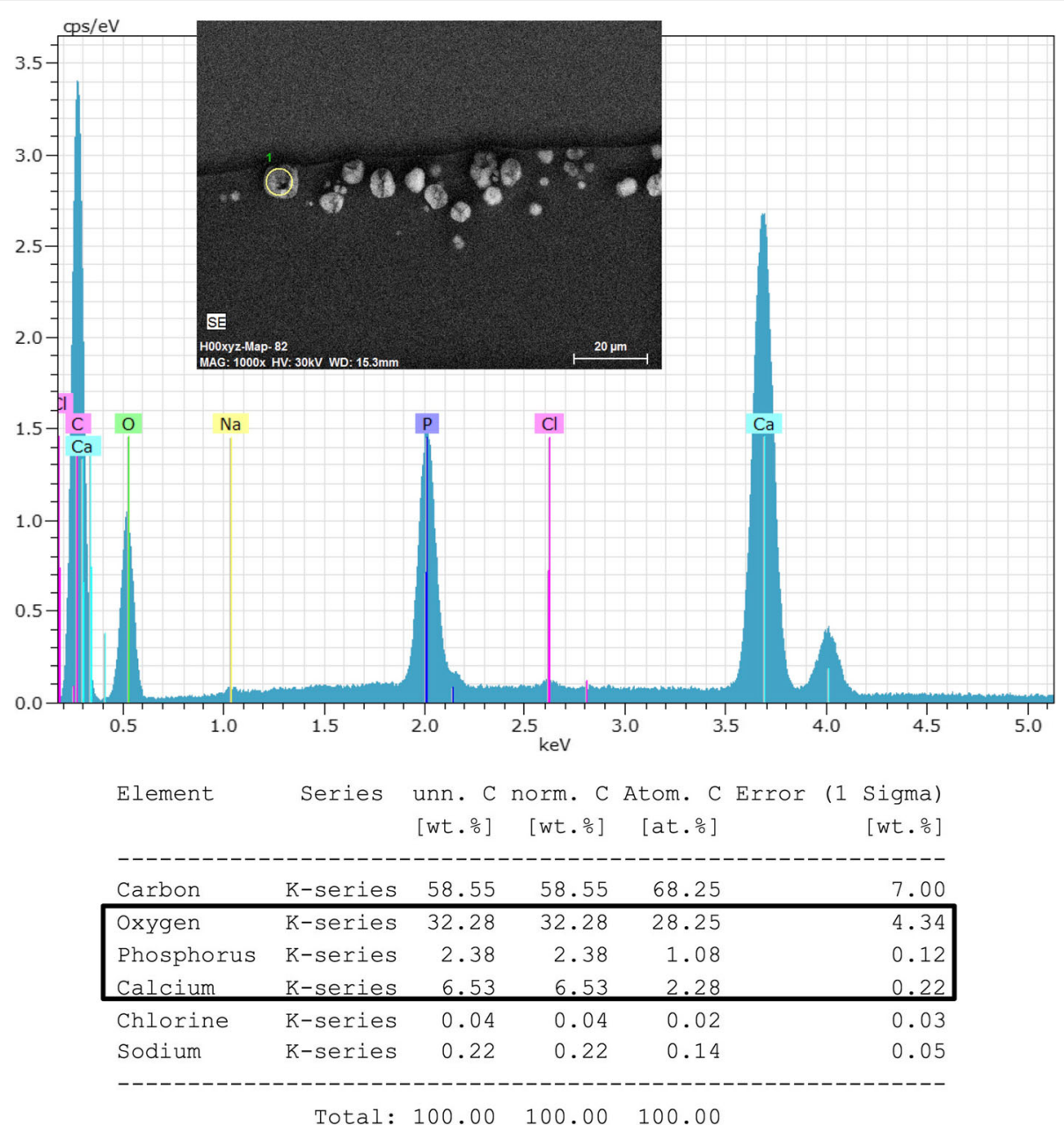

Fig. 5 Exemplary elementary analysis ( $(O L$ 5) using energy dispersive X-ray spectroscopy showing the deposits to consist of calcium phosphate

reports in the literature was also observed in procedures not involving air or gas instillation into the anterior chamber. Fung et al. report 7 cases of opacified hydrophilic IOLs after intracameral injection of rtPA to treat inflammatory fibrin membranes secondary to cataract surgery [5]. In 3 of these patients an IOL-exchange was performed. Similar to the results reported here, the laboratory analysis of the explanted IOLs showed calcium phosphate deposits on the surface/subsurface of the IOLs' anterior optical surface.

Different pathomechanisms have been suggested to play a role in hydrophilic IOL opacification after posterior lamellar keratoplasty and air or gas instillation into the anterior chamber. Metabolic changes in the anterior chamber due to the presence of the exogenous gas or other substances and an exacerbated inflammatory reaction caused by often multiple surgical procedures may lead to changes in the aqueous humor composition and cause the calcification of the IOL surface $[13,15,18]$. The supposed disturbance of the blood-aqueous-barrier caused by underlying conditions (mostly Fuchs' endothelial dystrophy) may amplify metabolic imbalance and intraocular inflammatory processes $[17,18]$. We believe, however, that the direct and prolonged contact of the IOL with the instilled exogenous gas, air or other substance is the key factor in the opacification of hydrophilic acrylic IOLs after posterior lamellar keratoplasty. The direct contact of the air or gas with the IOL possibly affects the IOL surface in a way that fosters the formation of crystallisation nuclei [10]. Dehydration of the hydrophilic acrylate material in contact with the exogenous air or gas may be one possible causative mechanism.

Especially in early stages of IOL-calcification, glistenings may be considered as an important differential diagnosis. In contrast to the opacifications in hydrophilic IOLs reported here, glistenings can occur in hydrophobic IOL materials. There are numerous reports in the literature on the formation of glistenings, especially in the AcrySof IOL (Alcon Laboratories, Fort Worth, Texas, 


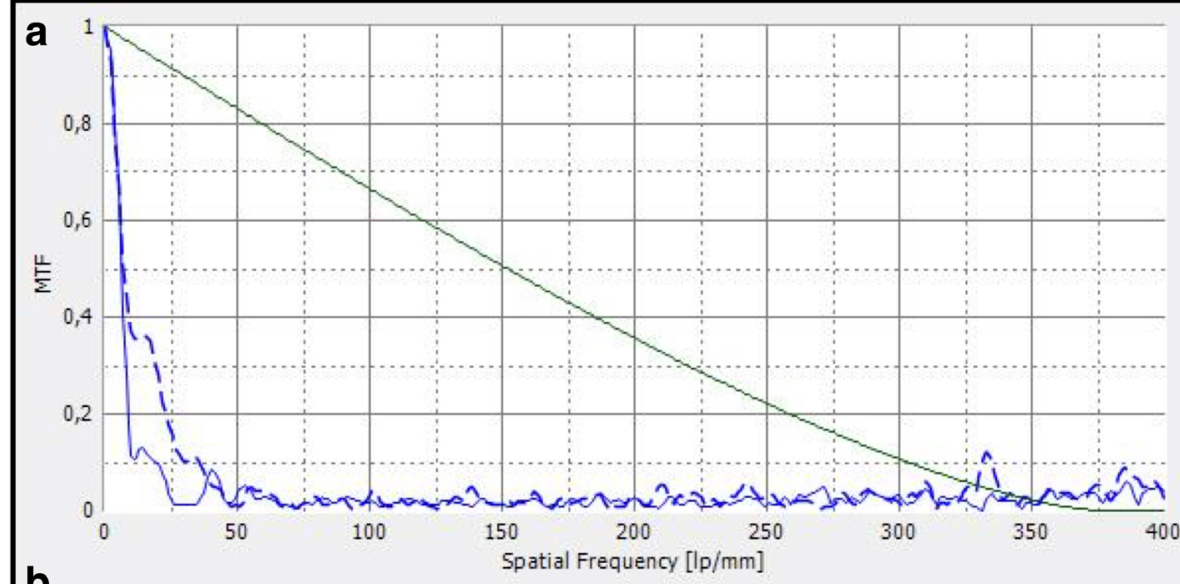

b
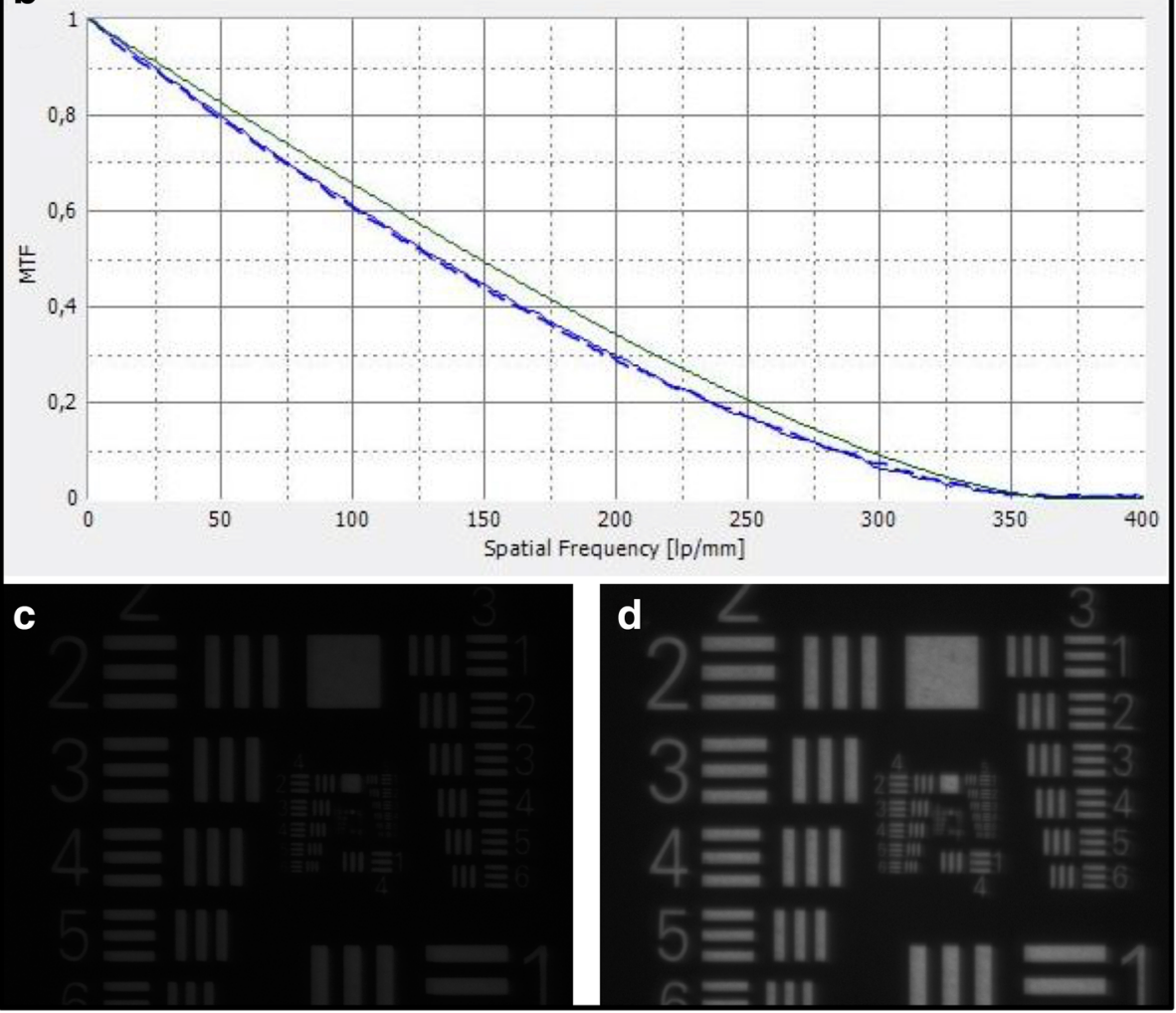

Fig. 6 (a) Representative modulation transfer function (MTF) of an opacified IOL (IOL 6) showing a deterioration at all spatial frequencies. The green line represents the diffraction limit. Blue lines represent the measured MTF values in sagittal (solid blue line) and tangential (dashed blue line) planes. (b) Representative MTF of a clear monofocal aspheric IOL from hydrophilic acrylate (CT Asphina 409 MP, Carl Zeiss Meditec, Jena, Germany) taken in our laboratory for comparison. (c) USAF target image obtained through IOL 6 at standard lighting conditions showing a significant reduction in light transduction. (d) With light adjustment an acceptable image quality could be achieved

USA) $[2,22,23]$. However, glistenings have also been observed in hydrophobic IOLs from other manufacturers [23]. Morphologically they correspond to microvacuoles within the hydrophobic IOL material. Although glistenings seem to reduce contrast sensitivity, a reduction of visual acuity is rare [23-26]. Aside from calcifications, other alterations of the IOL material have been described, such as IOL schisis, which is caused by the formation of a gap within the IOL material and may also reduce contrast sensitivity [27].

In addition to the laboratory analysis we assessed the optical quality of the calcified IOL explants by performing an optical bench analysis. This is the test method suggested in ISO 11-979-2 as the most suitable method for evaluating an IOLs optical properties. The ISO guidelines suggest that the system of model eye and IOL 
should exhibit an MTF value greater than 0.43 at $100 \mathrm{lp} /$ $\mathrm{mm}$. Our results clearly showed a deterioration of the optical quality with a marked decrease of the MTF across all spatial frequencies. Clinically this translates into a reduction of brightness and contrast especially in mesopic conditions and ultimately to „foggy "or "clouded "vision and loss of overall visual acuity. We have shown similar results in faulty manufactured IOLs in earlier publications [3,21], but to our knowledge this is the first systematic examination of calcified IOL explants after posterior lamellar keratoplasty using an optical bench in a larger series.

In symptomatic patients with decreased visual acuity caused by IOL calcification, an IOL explantation is the only treatment option. Previously, the incidence of IOL opacification/calcification was reported to be less than $1 \%$ [2]. However, with the advent of microinvasive keratoplastic techniques, reports about IOL opacifications and subsequent explantations have increased. Ahad et al. reported an incidence of 9.7\% IOL opacifications in 154 eyes after DSAEK [28]. Nieuwendaal et al. report an incidence of IOL opacification of 5\% in 160 eyes after DSEK with a $2.5 \%$ incidence of IOL exchange [29]. The explantation of IOLs involves the risk of intra- and postoperative complications. In a series of 25 eyes with opacified Aqua-Sense IOLs (a single-piece IOL from a hydrophilic acrylic copolymer with a water content of $25 \%$ by Aaren Scientific, Ontario, California, USA) Dagres et al. reported a complication rate (zonular dehiscence, posterior capsular rupture, corneal decompensation) of 48\% [14]. Especially a strong adhesion of the IOL to the capsular bag can make the explantation procedure challenging and in many cases an in-the-bag IOL implantation may not be possible. The indication for surgical removal of the opacified IOL therefore requires thorough preoperative diagnostic and informed patient consent and should only be considered in highly symptomatic patients. A Nd:YAG-Laser capsulotomy should not be performed in eyes with opacified IOLs, since it further increases the complication rate during an IOL exchange procedure. Obviously, a Nd:YAG-Laser treatment of the opacified IOL's surface, as has been intended in some cases (e.g. [13, 29]), will not be able to clear the opacifications that are situated underneath the optical surface and might in fact induce additional damage and further attenuate the IOL's optical quality.

\section{Conclusion}

IOL opacification is a rare postoperative complication. However, the instillation of air, gas or rtPA into the anterior chamber increases the risk of calcification for hydrophilic acrylic IOLs. This complication seems to be irrespective of the manufacturer. Since IOL explantation is associated with an increased intraoperative complication rate, it is recommended to avoid the use of hydrophilic acrylic IOLs in patients with endothelial dystrophy that will likely require procedures involving the intracameral instillation of air or gas, such as DMEK or DS(A)EK.

\section{Acknowledgments \\ The authors would like to thank Romain de Cock (Kent \& Canterbury Hospital, Canterbury, UK), John Dart (Moorfields Eye Hospital, London, United Kingdom), Günther Grabner (Paracelsus Medical University, Salzburg, Austria), Frank G. Holz (University Eye Hospital, Bonn, Germany), Dipak Parmar (Whipps Cross Hospital, London, United Kingdom) and Mark Whiting (St John of God Hospital, Geelong, Australia) for sending their IOL explants to the David J. Apple International Laboratory for Ocular Pathology.}

\section{Funding}

The International Vision Correction Research Centre and the David J. Apple International Laboratory for Ocular Pathology are supported by a grant from the Klaus Tschira Foundation. The funding source was not involved in any part of the study or the decision to submit the article for publication.

\section{Availability of data and materials}

The datasets used and/or analysed during the current study are available from the corresponding author on reasonable request.

\section{Authors' contributions}

BCG and TT contributed equally to this publication in analysing the data and writing the manuscript. GUA and RK conceived and designed the study. All authors were involved in data collection and analysis as well as drafting the manuscript. All authors read and approved the final manuscript.

\section{Ethics approval and consent to participate}

This study solely involves laboratory analyses of IOL explants. No additional procedures on humans or animals were performed. An informed consent and ethics committee approval were therefore not required.

\section{Consent for publication}

Not applicable.

\section{Competing interests}

None of the authors has a financial or proprietary interest in any material or method mentioned.

\section{Publisher's Note}

Springer Nature remains neutral with regard to jurisdictional claims in published maps and institutional affiliations.

\section{Author details}

${ }^{1}$ International Vision Correction Research Centre and the David J. Apple International Laboratory for Ocular Pathology, Department of Ophthalmology, University of Heidelberg, INF 400, 69120 Heidelberg, Germany. ${ }^{2}$ Department of Ophthalmology, Kangbuk Samsung Hospital, Sungkyunkwan University School of Medicine, Seoul, South Korea. ${ }^{3}$ Eye Clinic, Klinikum rechts der Isar der Technischen Universität München, Munich, Germany.

Received: 1 June 2017 Accepted: 14 August 2017

Published online: 22 August 2017

References

1. Werner L. Causes of intraocular lens opacification or discoloration. J Cataract Refract Surg. 2007;33(4):713-26. doi:10.1016/j.jcrs.2007.01.015. [published Online First: Epub Date]|.

2. Izak AM, Werner L, Pandey SK, Apple DJ. Calcification of modern foldable hydrogel intraocular lens designs. Eye (Lond). 2003;17(3):393-406. doi:10.1038/sj.eye.6700341. [published Online First: Epub Date]|.

3. Tandogan $\mathrm{T}$, Khoramnia $\mathrm{R}$, Choi $\mathrm{CY}$, et al. Optical and material analysis of opacified hydrophilic intraocular lenses after explantation: a laboratory 
study. BMC ophthalmology. 2015;15:170. doi:10.1186/s12886-015-0149-1. [published Online First: Epub Date].

4. Bompastor-Ramos P, Povoa J, Lobo C, et al. Late postoperative opacification of a hydrophilic-hydrophobic acrylic intraocular lens. J Cataract Refract Surg. 2016;42(9):1324-31. doi:10.1016/j.jcrs.2016.06.032. [published Online First: Epub Date].

5. Fung SS, Sykakis E, Islam NM, et al. Intraocular Lens Opacification following Intracameral Injection of Recombinant Tissue Plasminogen Activator to Treat Inflammatory Membranes after Cataract Surgery. J Ophthalmol. 2015;2015:975075 doi:10.1155/2015/975075 [published Online First: Epub Date]|.

6. Dhital A, Spalton DJ, Goyal S, Werner L. Calcification in hydrophilic intraocular lenses associated with injection of intraocular gas. Am J Ophthalmol. 2012;153(6):1154-60 e1. doi:10.1016/j.ajo.2011.11.017. [published Online First: Epub Date].

7. Fellman MA, Werner L, Liu ET, et al. Calcification of a hydrophilic acrylic intraocular lens after Descemet-stripping endothelial keratoplasty: case report and laboratory analyses. J Cataract Refract Surg. 2013;39(5):799-803. doi:10.1016/j.jcrs.2013.01.028. [published Online First: Epub Date]|.

8. Khan Ml, Muhtaseb M. Opacification of the intraocular lens implant following uneventful Descemet's stripping endothelial keratoplasty. Cont Lens Anterior Eye. 2011;34(2):92-3. doi:10.1016/j.clae.2010.12.002. [published Online First: Epub Date].

9. Patryn E, van der Meulen IJ, Lapid-Gortzak R, Mourits M, Nieuwendaal CP. Intraocular lens opacifications in Descemet stripping endothelial keratoplasty patients. Cornea. 2012;31(10):1189-92. doi:10.1097/ICO. 0b013e31823d1e12. [published Online First: Epub Date].

10. Schmidinger G, Pemp B, Werner L. Opacification of an intraocular lens: calcification of hydrophilic intraocular lenses after gas tamponade of the anterior chamber. Ophthalmologe. 2013;110(11):1066-8. doi:10.1007/s00347013-2793-2. [published Online First: Epub Date]|.

11. Neuhann IM, Neuhann TF, Rohrbach JM. Intraocular lens calcification after keratoplasty. Cornea. 2013;32(4):e6-10. doi:10.1097/ICO.0b013e31826150de. [published Online First: Epub Date].

12. Norouzpour A, Zarei-Ghanavati S. Hydrophilic Acrylic Intraocular Lens Opacification after Descemet Stripping Automated Endothelial Keratoplasty. J Ophthalmic Vis Res. 2016;11(2):225-7. doi:10.4103/2008-322X.158897. [published Online First: Epub Date].

13. Werner L, Wilbanks G, Nieuwendaal CP, et al. Localized opacification of hydrophilic acrylic intraocular lenses after procedures using intracameral injection of air or gas. J Cataract Refract Surg. 2015;41(1):199-207. doi:10. 1016/j.jcrs.2014.10.025. [published Online First: Epub Date].

14. Dagres E, Khan MA, Kyle GM, Clark D. Perioperative complications of intraocular lens exchange in patients with opacified Aqua-Sense lenses. J Cataract Refract Surg. 2004;30(12):2569-73. doi:10.1016/j.jcrs.2004.04.055. [published Online First: Epub Date]|

15. Maier PC, Heinzelmann S, Bohringer D, Reinhard T. Intraocular Lens Opacification Following Posterior Lamellar Keratoplasty. Klin Monbl Augenheilkd. 2015;232(8):976-81. doi:10.1055/s-0035-1546175. [published Online First: Epub Date]|.

16. Milojcic C, Latz C, Tandogan T, et al. Opacification of a hydrophilic acrylic intraocular lens after DMEK : A material analysis. Ophthalmologe. 2016 doi:10.1007/s00347-016-0394-6. [published Online First: Epub Date]|.

17. Morgan-Warren PJ, Andreatta W, Patel AK. Opacification of hydrophilic intraocular lenses after Descemet stripping automated endothelial keratoplasty. Clin Ophthalmol. 2015;9:277-83. doi:10.2147/OPTH.S78930. [published Online First: Epub Date].

18. Mojzis P, Studeny P, Werner L, Pinero DP. Opacification of a hydrophilic acrylic intraocular lens with a hydrophobic surface after air injection in Descemet-stripping automated endothelial keratoplasty in a patient with Fuchs dystrophy. J Cataract Refract Surg. 2016;42(3):485-8. doi:10.1016/j.jcrs. 2016.02.004. [published Online First: Epub Date].

19. Melles GR, Ong TS, Ververs B, van der Wees J. Descemet membrane endothelial keratoplasty (DMEK). Cornea. 2006;25(8):987-90. doi:10.1097/01. ico.0000248385.16896.34. [published Online First: Epub Date]|.

20. Price FW Jr, Price MO. Descemet's stripping with endothelial keratoplasty in 50 eyes: a refractive neutral corneal transplant. J Refract Surg. 2005;21(4):339-45.

21. Khoramnia R, Salgado JP, Auffarth GU, et al. Opacification of a hydrophilic intraocular lens 4 years after cataract surgery. A biomaterial analysis. Ophthalmologe. 2012;109(5):483-6. doi:10.1007/s00347-011-2487-6. [published Online First: Epub Date].
22. Moreno-Montanes J, Alvarez A, Rodriguez-Conde R, Fernandez-Hortelano A. Clinical factors related to the frequency and intensity of glistenings in AcrySof intraocular lenses. J Cataract Refract Surg. 2003;29(10):1980-4.

23. Werner L. Glistenings and surface light scattering in intraocular lenses. J Cataract Refract Surg. 2010;36(8):1398-420. doi:10.1016/j.jcrs.2010.06.003. [published Online First: Epub Date].

24. Dhaliwal DK, Mamalis N, Olson RJ, et al. Visual significance of glistenings seen in the AcrySof intraocular lens. J Cataract Refract Surg. 1996;22(4):452-7.

25. Xi L, Liu Y, Zhao F, Chen C, Cheng B. Analysis of glistenings in hydrophobic acrylic intraocular lenses on visual performance. Int J Ophthalmol. 2014;7(3):446-51. doi:10.3980/j.issn.2222-3959.2014.03.11. [published Online First: Epub Date]|.

26. Ogura Y, Ong MD, Akinay A, Carson DR, Pei R, Karakelle M. Optical performance of hydrophobic acrylic intraocular lenses with surface light scattering. J Cataract Refract Surg. 2014;40(1):104-13. doi:10.1016/j.jcrs.2013. 05.051. [published Online First: Epub Date]|.

27. Neuhann IM, Neuhann TF, Kleinmann G, Apple DJ. Clinical presentation of intraocular lens schisis. J Cataract Refract Surg. 2006;32(9):1503-8, doi:10.1016/j.jcrs.2006.04.016. [published Online First: Epub Date]|.

28. Ahad MA, Darcy K, Cook SD, Tole DM. Intraocular lens opacification after descemet stripping automated endothelial keratoplasty. Cornea. 2014:33(12):1307-11. doi:10.1097/ICO.0000000000000279. [published Online First: Epub Date]|.

29. Nieuwendaal CP, van der Meulen IJ, Patryn EK, Werner L, Mourits MP, Lapid-Gortzak R. Opacification of the Intraocular Lens After Descemet Stripping Endothelial Keratoplasty. Cornea. 2015;34(11):1375-7. doi:10.1097/ICO. 0000000000000574. [published Online First: Epub Date].

\section{Submit your next manuscript to BioMed Central and we will help you at every step:}

- We accept pre-submission inquiries

- Our selector tool helps you to find the most relevant journal

- We provide round the clock customer support

- Convenient online submission

- Thorough peer review

- Inclusion in PubMed and all major indexing services

- Maximum visibility for your research

Submit your manuscript at www.biomedcentral.com/submit
Biomed Central 